\title{
Fernando Birri: cine-poesía
}

\author{
Marco Franzoso ${ }^{\bullet}$ \\ Universidad Nacional del Litoral
}

\section{Créditos de apertura}

Creo que lo vimos todos, esa noche del 27 de diciembre, a ese señor muy viejo, despegar del suelo de Roma, abriendo sus alas enormes para partir a otro de sus viajes. Creo que todos los que estuvimos en contacto con Fernando Birri, ya sea personalmente o a través de su arte, tuvimos esa visión. Algunos quizás lo vieron salir disparado hacia la luna, cabalgando un cohete, un barrilete cósmico, entrar en órbita para seguir haciendo lo que mejor le sale: revolucionar. Lo vimos irse sonriendo, dejar este mundo hecho de realidad — «esa enemiga, esa perra flaca y gruñidora» (Birri, 1996: 15) — para entrar por fin y para siempre en el mundo de los sueños, medioambiente ideal para seres especiales, con alma de niño, barba larga y blanca, sombrero negro y alas enormes.

Este señor dejó la Tierra pero plantó semillas que florecieron, florecen y seguirán floreciendo para siempre. Sí, porque los que sueñan y los que hacen soñar logran engañar al tiempo con la poesía: en el caso de los sońadores, de los constructores de utopías, el cuerpo se va pero las ideas quedan y éstas, lo sabemos, revolucionan. Seguramente es por eso que Fernando Birri se acercó al cine, la más mágica de las artes, metáfora pura que nos muestra lo imaginado como si fuera real y, al mismo tiempo, nos puede enseñar lo real haciéndonos dudar de que lo sea. Porque, hay que decirlo, el cine fue sólo uno de los tantos mundos posibles en los que decidió desenvolverse este artista polifacético: poeta, titiritero, pintor, cineasta.

Es imposible catalogarlo, enjaularlo en las palabras. Algunos lo intentaron: «director», lo llamaron, «documentalista», «cineasta», «maestro», "padre del Nuevo Cine Latinoamericano», y además "comunista», "populista», «alocado», «traicionero». Él siempre se escurre de la definición, se despega la etiqueta. Hasta el nombre le queda chico y lo cambia: será Fermaghorg el Patafísico entre 1968 y 1978.

\footnotetext{
- Obtuvo el título de Laurea in Lingue e Letterature Europee, Americane e Poscoloniali de la Università Ca'Foscari Venezia (Italia) y de Licenciado en Letras de la Universidad Nacional del Litoral. Se desempeñó como docente en diferentes instituciones de Santa Fe y Paraná. Actualmente trabaja en la Cátedra de Italiano de la Facultad de Humanidades y Ciencias de la UNL y está desarrollando el Doctorado en Humanidades en la misma institución, cuyo tema de tesis es Fernando Birri. Sus ámbitos de investigación son: la literatura comparada, las relaciones culturales entre Italia y Argentina y el cine documental.
} 
Quizá la lengua no es un código apto para describir a Birri, quizá sería más fácil intentar hacerlo con la música o con la pintura. Quizás él mismo se definiría mejor así: caricatura — como las que diseñaba su padre—, máscara grotesca de sí mismo — como los personajes de Los inundados — o simplemente signo, mancha, fragmento de materia en un espacio infinito - como uno de sus fotoglifos.

Sin embargo, de todas las definiciones que se intentaron hacer, hay una que me parece la más atinada. Es la de Humberto Ríos, que dice de él: «es un artista del Renacimiento en el siglo XXI». Y ahora, pensándolo bien, me doy cuenta de que cuando veía sus fotos, sus videos, cuando leía sus textos, siempre me recordó a Leonardo Da Vinci: por sus "llovidas barbas de monje tibetano»" la inquietud, la energía creadora, la ansiedad de descubrir, de enseñar y, sobre todo, por un sueño en común con el genio toscano: poder volar. Después de la crítica y controvertida realización de la película $O R G$, Birri empieza a hablar de coSmunismo, es decir el comunismo cósmico. Con un guiño trasladó valores geo-locales a una dimensión galáctica pero recordó: "para hacer esto debemos por lo menos volar» (ibid: 237). Y para volar no son necesarias maquinarias complejas, sino simplemente las ganas de hacerlo, la voluntad de vivir la utopía.

Un hombre, Birri, cuya obra maestra es, sin duda alguna, su propia vida y cuya preocupación principal fue la de seguir ( $\mathrm{r}$ )evolucionando: «una revolución que no revoluciona permanentemente sus lenguajes, alfabetos, gestos, miradas, involuciona o muere» (ibid: 235), escribió.

Humanista contemporáneo, puso su arte al servicio del hombre: la cámara fue ojo, el micrófono oído y las palabras cerebro. «Cada error en la interpretación del hombre, comporta un error en la interpretación del mundo» se lee en su manifiesto Por un Nuevo Nuevo Cine latinoamericano (ibid: 236).

En estas pocas páginas trataremos de tejer unas líneas para leer la vida-obra de Fernando Birri, focalizando la atención en la relación fundante entre cine y poesía. Conscientes de que se trata de una simple aproximación, se escavará en lo que menos se difundió sobre el autor; recuperaremos material quizás secundario pero útil para nuestro montaje, no siempre cronológicamente lineal. De hecho la memoria no es otra cosa que un proceso de selección de recuerdos montados y ordenados con el fin de construir una idea de vida. Y la persona en cuestión, según Galeano, «tiene más de siete vidas y miles de lenguas, responde al nombre de Fernando Birri, pero se llama también con todos los nombres de la gente que se lleva adentro" (Birri en De Pascale, 1994: 5)².

En este trabajo se usarán como fuentes principales textos de su autoría, clases, entrevistas y discursos públicos. Confiaremos en sus palabras, conscientes de que a los locos, a los artistas y a los magos no siempre se les puede pedir cohesión y coherencia; pero también sabiendo que nadie analizó la vida y la obra de este artista como lo hizo él a lo largo de más de ochenta años de carrera.

\section{Primera parte: en el principio fue la poesía, raíz de todas las flores}

Nieto de un molinero y anárquico italiano, emigrado por hambre y política desde los campos del Friuli Venezia Giulia a la Pampa Gringa, hasta donde terminaba el ferrocarril. El 
padre heredó del abuelo la pasión por la pintura y la simpatía política, no obstante el salto de clase. De hecho Fernando Birri padre, escribano de profesión, frecuentó una escuela de arte y fue un gran caricaturista y crítico — publicó sus artículos en el diario El Litoral. Todos los hermanos del padre eran también pintores y músicos. Ramón —el ídolo de Fernando—, Valerio, Massimo y Desiderio Birri formaron el Cuarteto típico Birri y navegaron río arriba el Paraná hasta llevar por primera vez el tango al Paraguay.

El niño Fernando Birri creció entonces en un entorno cultural y artísticamente favorable, donde pudo desarrollar las artes plásticas y la escritura. Birri recuerda la máquina de escribir del padre, y su primer poema, escrito a los cuatro o cinco años, dedicado a un primo llamado Horacio Pierrout: "Y entonces vino mi primo Pierrú ly la vaca dijo muиuuu! Fue mi primera obra maestra» (Birri, 2007: 344).

A través del padre alimentó también la curiosidad hacía el cine. Con él iba los domingos a la función vermouth del cine Colón, vio la primera película sonora, grabó sus primeros $8 \mathrm{~mm}$ reproducidos en unas sábanas colgadas en el living de casa.

Escapando de la siesta obligada, se iba a leer los libros prohibidos en la biblioteca de familia: de Verne a Rivera, de Huasipungo a Doña Barbara, pero también —más adelante-Il Manifesto futurista de Marinetti, El arte y la vida social de Plejanov, El arte y la locura de Vinchon. Estos textos, que marcaron profundamente la manera de concebir el arte de Birri, desdibujan un linaje artístico y, sobre todo, de ideales políticos y éticos que empieza con el abuelo y siguen con el padre para llegar a él.

En la adolescencia la lectura se hizo necesidad exasperante, junto con el placer de la escritura. El descubrimiento de Federico García Lorca fue la llave de entrada a un «mundo sensual, caluroso, misterioso, inquietante, vibrante, excitante» (Birri en De Pascale, 1994:13). Lorca fue para él un escritor mágico, un amigo con el cual convivió muchos años y a través del cual aprendió que se puede hacer de una vida una gran metáfora, separándose de sí mismo.

Cuando en 1949 Birri conoce a Rafael Alberti en Buenos Aires, éste le confiesa: «la bala que mató a Federico estaba destinada a mí» (ibid: 21). Desde ese momento en adelante se instauró una amistad y complicidad entre los dos artistas que llevaría a varias colaboraciones y a una convivencia en el mismo barrio de Roma, forzada por el exilio común. En Rafael Alberti. Retrato de un poeta ${ }^{3}$ (1983) se retoma esa frase pronunciada por el artista espańol treinta años antes, el cual se "confiesa» frente a la cámara explicando que efectivamente, si alguien tenía que morir de esa manera tenía que ser él, más comprometido políticamente. En la película está presente el «ectoplasma de Lorca» (ibid: 21) que, a través de la voz de Alberti habla del Juego y la teoría del duende, según la cual:

La poesía de todos los tiempos proviene de tres fuentes de inspiración: la musa, que da la poesía clásica; el ángel, que da una poesía innovadora con un encanto refinado y que se identifica fundamentalmente con el Renacimiento y la Edad de Oro de la poesía española; y el duende, del que viene la poesía que tiene sus raíces en lo popular (Birri, 2007: 180)

Alberti y Lorca son, para Birri, poetas «del ángel» y han sabido, cada uno a su manera, transformar sus valores artísticos en valores políticos. En el mismo plano afectivo-intelectual está Neruda, otra lectura que acompañó a Birri para toda la 
vida y otra persona — poeta en carne y hueso- que se convertiría en un confidente durante el segundo exilio italiano del autor.

Canto general, Livre de chevet de Birri es, en palabras del autor «la historia de América Latina en verso» (ibid: 335). Neruda encarna una dimensión regional (chilena) y, al mismo tiempo Latinoamericana. Es el prototipo del intelectual-poeta latinoamericano, creador de un lenguaje regional y universal al mismo tiempo: un lenguaje de pasión que se opone al lenguaje de la razón europeo. Subraya Birri en una clase:

Si se piensa en los poetas de América Latina, en Neruda, Rubén Darío, Gabriela Mistral, Alfonsina Storni, José Martí, lo que encontramos es un lenguaje impregnado de un sentimiento tan fuerte que se impone al raciocinio. No es que sea un lenguaje infrarracional: es metarracional, va más allá; no es que niegue la razón sino que la sublima y pasa a una categoría superior del espíritu humano, de la inteligencia humana incluso, de la cual la razón es una parte pero no la principal ni la esencial. (Ibid: 335)

La búsqueda de un «lenguaje latinoamericano» es una preocupación que marca los intereses de Birri desde sus primeros pasos. Esta necesidad, que une a muchos artistas del continente sobre todo a partir de los años ' 60 , trasciende los límites nacionales y también las fronteras entre textos diferentes. Exponentes de la literatura, del cine, del teatro y de las artes plásticas se reconocen en una red de ideas comunes sobre cómo debería pensarse el «arte latinoamericano».

El Nuevo cine latinoamericano, movimiento del cual Birri es considerado justamente el padre, tiene como objetivo principal la creación de una estética fílmica latinoamericana: un cine desde el subdesarrollo que no se haga cómplice del subdesarrollo, lo define Birri; «cine imperfecto» para el cubano J.G. Espinoza; una «estética del hambre» según Glauber Rocha; un «tercer cine» para Getino y Solanas.

El poeta que cierra la tríada indispensable para Birri es Walt Whitman que «pertenece a Manhattan, después a los Estados Unidos y al continente americano» (Birri en De Pascale, 1994: 33). Con su introspección proyectada hacia los otros, Whitman representa «lo nuevo» de América toda.

Lorca, Neruda y Whitman fueron tres maestros, tres ejemplos de libertad y creación expresiva. Birri se nutrió de ellos, los digirió y asimiló convirtiéndolos en parte de él. En esto está la fórmula alquímica que define al autor: arte-sociedad-locura.

Durante su primera estadía italiana, para estudiar en el Centro Sperimentale de Cinematografía de Roma, no encontró esa locura. En la capital mundial del clasicismo, el joven Birri no encontró poética vanguardista que caracterizaba «el artista latinoamericano», sino una poesía más rigurosa y anclada a la realidad. Leyó a Quasimodo, a Montale, a Pavese, a Pasolini, pero eran mucho más prosaicos que toda la poesía a la que él estaba acostumbrado. Sin embargo, en la Italia neorrealista descubrió la poesía en el cine: «los poetas italianos de la posguerra fueron los cineastas y los narradores de lo cotidiano» (ibid: 40). La sumersión en el mundo cinematográfico italiano influenció enormemente las ideas estéticas de Birri, que poco a poco se dio cuenta de las posibilidades revolucionarias del cine. De hecho, en la base de la estética neorrealista no estaba simplemente la idea de representar la situación —a menudo trágica — de la Italia de posguerra, sino también un impulso transformador: representar la realidad para cambiarla. Este impulso, revolucionario para cualquier movimiento artístico, es la chispa que trajo Birri de Italia a Santa Fe, donde fundó el Instituto de cine de la UNL, pero esa es otra historia. 


\title{
Segunda parte: arte-sociedad-locura, una fórmula alquímica
}

\author{
"Cada error en la interpretación del hombre, \\ comporta un error en la interpretación del Universo y de la Historia, \\ y es por lo tanto un obstáculo a su transformación». \\ Actualización de una antigua fórmula alquímica.
}

Como vimos en el apartado anterior, la poesía cumple una función fundante en la "visión del mundo» de Fernando Birri, el cual desde siempre escribió poemas y trató de trabajar en la frontera entre cine y poesía. Explica en una clase:

Aquí tenemos que distinguir, para entendemos, entre poesía con «P» mayúscula y poesía con «p» minúscula (...) Hablo de algo que ya les dije en otras lecciones anteriores, poiesis, creación en el sentido más amplio. Pero si ésta es la poesía con mayúscula, que se manifiesta en todas las formas de la creación — porque en la arquitectura de un templo griego o de una pirámide maya hay una forma de la poesía, una expresión de la poesía al igual que en la música o en cualquier forma de arte- también está la poesía con p minúscula, el delicado arte de hacer versos como una forma específica de la poesía escrita.

En toda la concepción de mi cine y en mi manera de ver la obra de arte, creo que ha sido predominante esta visión poética de la creación artística. (Birri, 2007: 344)

Birri nace poeta y no dejará nunca de serlo. Su primer libro de poemas Cuaderno de Bitácora. Autorretrato del otro lo escribió mientras trabajaba como ayudante de cocina en un barco en el Río Paraná. Este texto, publicado en una magnifica edición de 2008 por Casa de las Américas, demuestra como el tema del «otro» estaba muy presente en el Birri adolescente.

El «otro» que está también representado por el sincretismo (socio-cultural o ideológico-político) que caracteriza a América Latina. El cine - pero también la poesía - de Birri nace y se desarrolla a través de lo que él mismo define "pensamiento aluvional», o sea la idea de que:

En el latinoamericano se conjugan una serie de dimensiones del pensamiento que son el resultado de nuestra propia historia [...] Por un lado tenemos las raíces que [...] corrieron por debajo de la tierra y ahora han vuelto a brotar. [...] Por el otro lado tenemos el pensamiento inmigratorio [...] guerrero, con la cruz y la espada y la sífilis coloniales, o del pensamiento inmigratorio pacífico que, [...] vino con arados pero también con una filosofía del hambre y una libido revolucionaria de justicia y, contradictoriamente, una libido conservadora de ahorros pequeño-burgueses. Todo se junta en nosotros [...]» (Birri, 2007: 19)

El autor pone en práctica este pensamiento, cumpliendo un trabajo de síncresis, cultural y estilística. Sus obras manifiestan la voluntad de superar las barreras y los límites formales: poesía, literatura, sociología y antropología entran en el cine; las fronteras entre ficcional y no ficcional se suprimen.

Este Birri es "Uno, nessuno e centomila» ${ }^{4}$ y en su arte no hay que encontrar continuidades, sino pensarla como un "caleidoscopio de formas expresivas que, 
en la especificidad de sus lenguajes de espejos y ecos, dan vida a una armonía del discurso" (Birri en De Pascale, 1994: 91).

Esta creatividad sin fronteras está, sin embargo, siempre direccionada hacia revolución: de las formas, del lenguaje, de la sociedad. Arte activo: cambiante y arte que cambia. Por esto, entendemos que se pueda trazar un hilo conceptual-ideológico entre dos textos que marcan momentos claves de la carrera de Fernando Birri: el manifiesto un «Cine nacional, realista y crítico» (1958) y «Un cine cósmico, delirante y lumpen» (1978)

El primer manifiesto sigue el estreno de Tire Dié (primera encuesta social filmada del país). Este documento, fruto de la experiencia del Instituto de Cine de Santa Fe es testimonio de una de las experiencias cinematográfica más revolucionarias de la historia de América Latina, no sólo por ser la primera escuela de cine argentina, sino también porque marca una tendencia en la concepción de la obra de arte como arma para la emancipación de la colonización cultural norteamericana y europea. La búsqueda de una identidad nacional, problemática constante de los países de América Latina, encontraría solución entonces solamente a través de una mirada histórica, realista y crítica. El manifestó subraya la necesidad «moral» de reformar el cine nacional, aportando problemáticas sociales inéditas a través de una actitud crítica. En el punto tres leemos:

Utilizar el cine al servicio de la Universidad y la Universidad al servicio de la educación popular. En su acepción más urgente esta educación popular va entendida como toma de conciencia cada vez más responsable frente a los grandes temas y problemas nacionales, hoy y aquí. (Birri, 2007: 231)

La identidad nacional (no nacionalista) se encontraría entonces no tanto en las características peculiares del país y, menos aún, en las diferencias con los otros, sino más bien en una condición común a todos los países de América Latina (y de todos los «sur» del mundo). Lo nacional se transforma entonces en una herramienta de cohesión entre los países, en un arma continental desde el subdesarrollo y en contra del subdesarrollo.

El segundo documento mencionado («Para un cine cósmico, delirante y lumpen») acompañó la salida de la película $O R G$ y fue la culminación de un periodo particular y duro de la vida de Fernando Birri, después de su segundo exilio en 1963. Después de dejar Argentina emprendió un largo peregrinaje a lo largo de América Latina, donde no encontró situaciones favorables, así que tuvo que volver a Italia. Ahí vivió una crisis que lo llevó a trabajar por diez años en el montaje de la película $O R G$, película-experimento, «un no-filme», "un cine de loco para locos», "anti-película», provocativo trabajo de collage que juega con la percepción y los sentidos del espectador. Este nuevo exilio interior obliga el autor a confrontarse con sí mismo y con la sociedad que lo rodea. La realidad lo había desilusionado y buscó refugio en los mundos oníricos que el cine permite construir. Birri decidió alejarse, alienarse de la realidad social, cultural, política. De esta experiencia nació la idea de coSmunismo, de comunismo cósmico, explicitada en el manifiesto que acompañó la salida de la película: 
... comunismo cósmico y mágico por un cine cósmico delirante y lumpen totalmente discutible por sus métodos y tiempos de rodaje y de montaje (pero toda la operación es una demostración de que se puede poner en práctica la Utopía) locura y rigor tomados de la mano no habrá revolución duradera sin revolución del lenguaje tabula rasa (ibid: 83)

Estas ideas aparentemente distantes de las presentadas en el manifiesto del 1958 son en realidad su consecuencia y ampliación. La dimensión "cósmica» está directamente relacionada a la «nacional» si se toma en consideración lo mencionado anteriormente: lo nacional desde una perspectiva histórica y crítica une y no divide. Lo crítico y popular se transforma en «lumpen», es decir marginal radicalizado. De cine realista se pasa a hablar de cine delirante: no hay diferencia entre un realismo construido (por la historia oficial) y el delirio. De hecho ¿̨no es acaso la realidad un gran juego de magia?

\section{Créditos de cierre}

Los dos textos mencionados nos muestran dos de las miles caras de Fernando Birri: artista y hombre en continuo diálogo consigo mismo, discurso de cientos de voces, contradictorio, hijo pos-moderno del «siglo del viento».

Después del (auto)exilio de $O R G$, Birri se volvió a poner su máscara de hombre público: además de seguir con su actividad como director, emprendió proyectos de difusión del cine y cultura latinoamericanos. "Gracias a él América Latina ya no nos parece tan distante» (Fasoli, 1988: 10) ${ }^{5}$, subrayó el crítico Doriano Fasoli. Birri aplicó a su vida y a su trabajo valores universalistas, achicando distancias a través de lenguajes comunes; fundó escuelas, dictó clases y seminarios haciendo propia la "poética de la didáctica» que según Tarik Souki lo caracteriza.

En Birri vida, arte y política se fundieron en esa alquimia que hacen de un hombre un símbolo. Como el propio Souki subraya:

Hay obras y vidas como las de Fernando que aparecen señaladas, en extensión y en profundidad, por principios inequívocos de espiritualidad, y que en mi concepto lo marcan ser humano auténtico y pleno, esto es: esencia, ser, totalidad, de poeta. (Souki, 2011: 106)

«Ya no se más dónde empieza la palabra cine y dónde termina la palabra vida, tampoco sé más dónde termina la palabra poesía y donde empieza la palabra revolución» (Birri, 1996: 236). 


\section{Notas}

${ }^{1}$ Fragmento del poema «Fernando Birri» de Rafael Alberti (Madrid, Otoño de 1983)

${ }^{2}$ Todas las entradas citadas como «Birri en De Pascale» son traducciones personales.

${ }^{3}$ «Rafael Alberti. Retrato de un poeta» es una película documental de 1983 dirigida por Fernando Birri

${ }^{4}$ «Uno, nessuno e centomila» es una novela publicada en 1925 por Luigi Pirandello. En la novela se enfrenta el tema del relativismo en la relación del individuo consigo y con el «otro». El individuo es un sujeto fragmentado en la mirada del «otro» y es al mismo tiempo uno, nadie o cien mil. ${ }^{5}$ Traducción personal.

\section{Referencias bibliográficas}

Birri F. (1996) El alquimista democrático. Por un nuevo nuevo nuevo Cine Latinoamericano. Santa Fe: Ediciones Sudamérica

(2007) Soñar con los ojos abiertos. Las treinta lecciones de Stanford. Buenos Aires: Aguilar, Altea, Taurus, Alfaguara.

De Pascale G. (1994) Fernando Birri. L'Altramerica. Pompei (Napoli): Le Pleiadi

Fasoli D. (1988) Fernando Birri. Il nuovo cinema latinoamericano. Roma: Ediz. Associate

Souki T. (2011) «Una poética de la didáctica (Sobre Fernando Birri)» en Doc On-line n. 10 (pp.105-152) www.doc.ubi.pt

\section{Franzoso, Marco}

«Fernando Birri: cine-poesía». El hilo de la fábula. 